\title{
Adaptive tracking and recording method for dynamic real-time WiFi
}

\section{fingerprint positioning}

\author{
Liang Dong, Dong Liang \\ Beijing University of Posts and Telecommunications Beijing, China I.dong@qmul.ac.uk \\ Email: 1105316190@qq.com
}

Key Words: WIFI, Fingerprint Positioning, Adaptive Tracking, Internet of Things, Algorithm

\begin{abstract}
The overall performance of fingerprint positioning system will be improved in this paper with the adoption of technology in artificial intelligence field and optimization technology. First, at offline stage, adaptive tracking and recording method for dynamic real-time WiFi fingerprint positioning has been proposed. Regional propagation model (RPM) of indoor wireless signal has been proposed through the RSS value clustering fading characteristics observed based indoor zoning principle in clustering channel modeling algorithm. Take samples from the reference points of sparse density distribution with adoption of affinity propagation clustering technology to attain fingerprints, and then divide the indoor area into sub regions with specific number, make use of the sampling data of each sub region to set up proposed RPM path loss propagation model and predict the fingerprints of other unmeasured reference points through this model to reconstruct complete fingerprint database. Experimental testing results have shown that the RSS prediction accuracy of proposed path loss propagation model is higher than existing propagation model; at the same time, when decrease fingerprint acquisition workload above $28 \%$, the proposed algorithm can still attain high positioning accuracy.
\end{abstract}

\section{Introduction}

Federal Communications Commission (FCC) published E911 order in 1996, which promotes the development of wireless positioning technology greatly. In recent years, wireless positioning technology starts to be applied in design, optimization, maintenance and other aspects of cellular communication system. How to provide effective and accurate positioning system without increasing system burden has become the field research focus. At present, main researches on wireless positioning methods both at home and abroad include CELLID positioning method, circumferential positioning method, TOA/TDOA positioning method and fingerprint positioning method etc, in which the fingerprint positioning method is with great potentiality in accuracy and has become the research focus.

In the research on fingerprint positioning, literature [1] studies fingerprint positioning algorithm based on spatial segmentation, divides the positioning region into grids, saves latitude and longitude values of $\mathrm{i}$ base stations with the closest distance to each grid center, sets up corresponding fingerprint database and attains the position coordinates of positioning points, but the establishment of fingerprint database in literature [1] does not consider about the practical wireless environment. Literature [2, 3] study fingerprint algorithm based on propagation model modification RSS, make use of the modified RSS to set up fingerprint database, attain the position coordinates of positioning points after comparing the fingerprints and increase the computational complexity of algorithm. 


\section{Principle of fingerprint positioning}

Fingerprint positioning originates from Database Correction Method (DCM) [4] and it can be applied to any cellular network. Figure 1 is fingerprint positioning system model, including data preprocessing [5], establishment of fingerprint database and fingerprint comparison. Fingerprint database can be set up based on signal characteristic parameters of mobile station at different positions. Fingerprint comparison has been made for the signal characteristics of positioning points and signal characteristics stored in database to realize the positioning of mobile station.

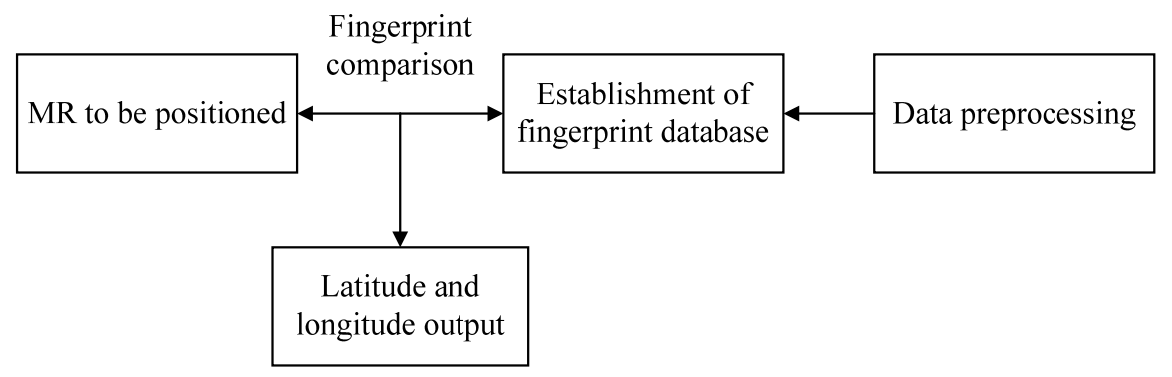

Figure 1 Fingerprint Positioning System Model

Fingerprint database technology makes use of multipath effect effectively and combines multipath and position information together. In the region with small wireless environment change, as the multipath structure of information channel is with uniqueness at the same position, this kind of multipath structure can be taken as fingerprint [6]. In communication process, mobile station sends out one measurement report (MR) frame to base stations (BTS) in every 480s (470ms at signaling channel). Study and elaborate MR frame through Abis port in real time to attain the RSS values of small main service cell and six strongest neighboring cells. At this moment, RSS can be taken as the characteristic signal of one position and then realize fingerprint positioning.

\section{RSS adaptive tracking}

Okumura-Hata model can be expressed with formula (1):

$L=K_{1}+K_{2} \log +K_{3} \lg d+K_{4} \lg h_{b} \lg d+K_{5} \lg h_{m}+\mathrm{A}\left(h_{m}\right)$

In which, $\mathrm{L}$ is power loss, attained from decreasing base station power from RSS; $K_{1} \ldots K_{5}$ are coefficients to be modified; $\mathrm{f}$ is the working frequency band of radio wave; $\mathrm{d}$ is the distance between sending and receiving antennas; $h_{b}$ is the effective antenna height of base station transmitter (relative sea level height of base station deducting the average terrain height between $3 \mathrm{KM}$ and $15 \mathrm{KM}) ; h_{m}$ is the effective antenna height of mobile station receiver and $\mathrm{A}\left(h_{m}\right)$ is modification factor of mobile station antenna.

In Okumura-Hata model, ${ }^{\mathrm{A}}\left(h_{m}\right)$ relates to height and density of buildings in practical wireless environment as well as antenna gain etc. The deviation of modified propagation model [7] will increase the RSS error and the algorithm will be very complicated.

Because RSS in the same service cell is with spatial similarity, spatial segmentation method has been applied to process data region and K-means algorithm [8] has been used to make clustering for RSS of each grid. Clustering with more samples is normal sample, vice versa. As noise point, set its RSS as missing value. After meaning normal samples, repair default values and finally make mean value filtering based on temporal correlation.

Segmentation and clustering

Based on the largest and smallest latitude and longitude values, spatial segmentation has been made for the region and the region has been divided into $n_{X} * n_{Y}$ grids. Assume the longitude range of grid is $\left(\operatorname{Lon}_{\min }, \operatorname{Lon}_{\max }\right)$ and latitude range is $\left(\operatorname{Lat}_{\min }, \operatorname{Lat}_{\max }\right)$, set the longitude and latitude values of data as 
(Lon,Lat), and

$$
\begin{aligned}
& \operatorname{Lon}_{\text {min }}<\text { Lon }<\text { Lon }_{\text {max }} \\
& \text { Lat }_{\text {min }}<\text { Lat }_{\text {Lat }}<t_{\text {max }}
\end{aligned}
$$

Classify this data point into this grid and information included in this point is LAC, CELL_ID, RSS and longitude and latitude values measured by GPS. K-means has been adopted to make clustering for each grid non-empty value:

(1) Select $\mathrm{K}=2$, assume initial clustering center is ${ }^{\left(x_{0}, x_{1}\right)}$, number of samples is $\mathrm{n}, \quad\left(x_{0}, x_{1}\right)$ is the minimum and the maximum RSS, that is:

(2) For each sample $X_{i}(1<=i<=n)$, compare the Euclidean distance between $X_{i}$ and ${ }^{x_{i}}$, divide $X_{i}$ into the closest clustering $C_{i}$ and form into initial clustering.

(3) Recalculate the center ${ }^{c_{i}}$ of each clustering in step 2 and update the center of each clustering $C_{i}$.

$$
c_{i}=\frac{1}{m} \sum_{i=1}^{m} X_{i}
$$

(4) Recycle (2) to (3) until standard measurement function starts to converge.

$$
D=\sum_{i=1}^{k} \sum_{j=1}^{n}\left|X_{i}^{(j)}-c_{i}\right|
$$

Time smoothing

After clustering, set the RSS of noise point at empty and mean N RSS in normal sample.

$$
\text { Level }_{\text {aver }}=\frac{1}{N} \sum_{i=1}^{N} \text { Level }_{i}
$$

In which, Level $_{\text {aver }}$ is the mean of N RSS, Level is one RSS in the sample and use Level $_{\text {over }}$ to replace each empty value; finally, make time smoothing for RSS.

$$
\text { Level }=\frac{\text { Level }_{t-1}+\text { Level }_{t}+\text { Level }_{t+1}}{3}
$$

In which, Level is the level value after smoothing at one moment; Level $t_{t}$ is the level value needs to be smoothed at one moment; Level $_{t-1}$ is the level smaller than this moment and belongs to the same cell with Level $_{t}$; Level $_{t+1}$ is the level bigger than this moment and belongs to the same cell with Level $_{t}$.

\section{Establishment of fingerprint database}

MR frame analysis is the key step for signal fingerprint extraction and fingerprint database establishment. Analyze MR frame in real time through Abis interface between base station and base station controller. Analyze and extract effective field information for mobile station positioning and take it as fingerprint reference. Screen the message type of road measurement data and set up corresponding fingerprint database. Figure 2 is MR frame analytic example. 


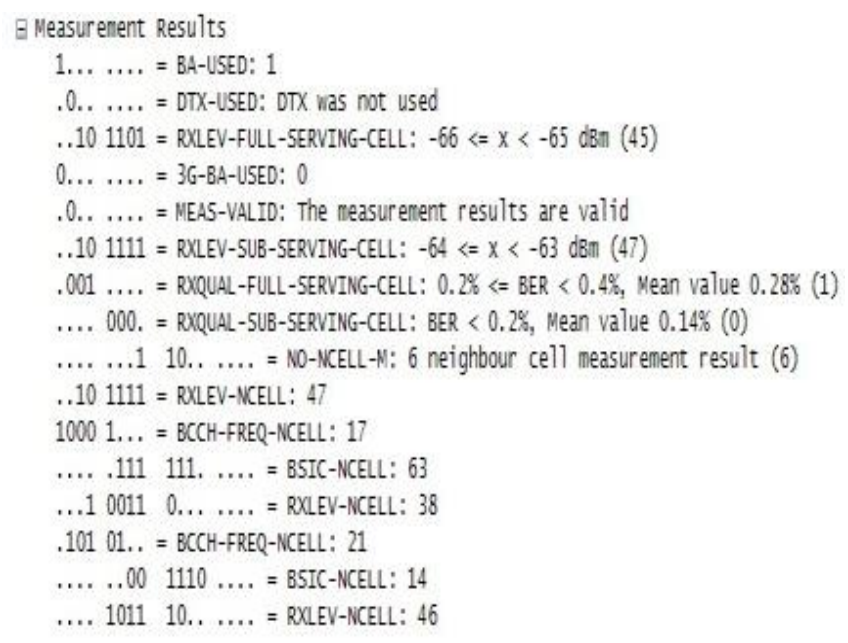

Figure 2 MR frame analytic example

Effective field information can be attained after making MR frame analysis:

(1) Location area code (LAC) and CELL_ID of main service cell and six strongest neighboring cells.

(2) Received signal strength of main service cell and six strongest neighboring cells.

(3) Broadcast control channel $(\mathrm{BCCH})$ carrier, network color code $(\mathrm{NCC})$ and base station color code (BCC).

Extract data whose road measurement data message type is MR and analyze cell information based on above effective field storage data. After making RSS space time process for main service cell and six neighboring cells, make use of latitude and longitude values measured by LAC, CELL_ID, RSS and GPS of each cell to set up fingerprint database.

\section{Fingerprint comparison}

For MR needs to the positioned, screen data with the same service cell as MR in fingerprint database, attain several fingerprint data bigger or equal to 1 with this MR (number of the same neighboring cell), make RSS vector distance comparison and then attain the longitude and latitude values of positioning point.

Set the characteristic vector composed of RSS of MR as $V_{1}=\left(\right.$ Level $_{m r_{-} 0}$, Level $_{m r_{-} 1}, \cdots$, Level $\left._{m r_{-} 6} 6\right)$, screening characteristic vector from fingerprint database as $V_{2}=\left(\right.$ Level $_{\text {prin__ }}$, Level $_{\text {print__}}, \cdots$, Level $\left._{\text {print_6}}\right)$ and then the Euclidean distance between $V_{1}$ and $V_{2}$ is:

$$
D=\sqrt{\sum_{i=0}^{6}\left(\text { Level }_{\text {mr } \_i}-\text { Level }_{\text {print_i }}\right)^{2}}
$$

Select the number of same neighboring cell ${ }^{I=J(0<=J<=6)}$; many similarity points corresponding to I value can be screened out from fingerprint database. Based on K-Nearest Neighbor (KNN), calculate the Euclidean distance D between similarity point RSS and RSS of MR needs to be positioned. Order D from big to small and select the smallest longitude and latitude values of first $\mathrm{N}$ similarity points of $\mathrm{D}$ to mean and attain positioning result. Analyze the error of positioning result Error $_{i}(0<=i<=6)$ and attain reasonable selection method for I value.

\section{Results analysis}

In the experiment, two test mobile stations have been adopted as calling and called. LOG file of data has been stored along specific grid, road test data line is as shown in Figure 3. After a period of time, select some routes to make road test again. Experimental data can be attained through 
analyzing LOG file of corresponding data.

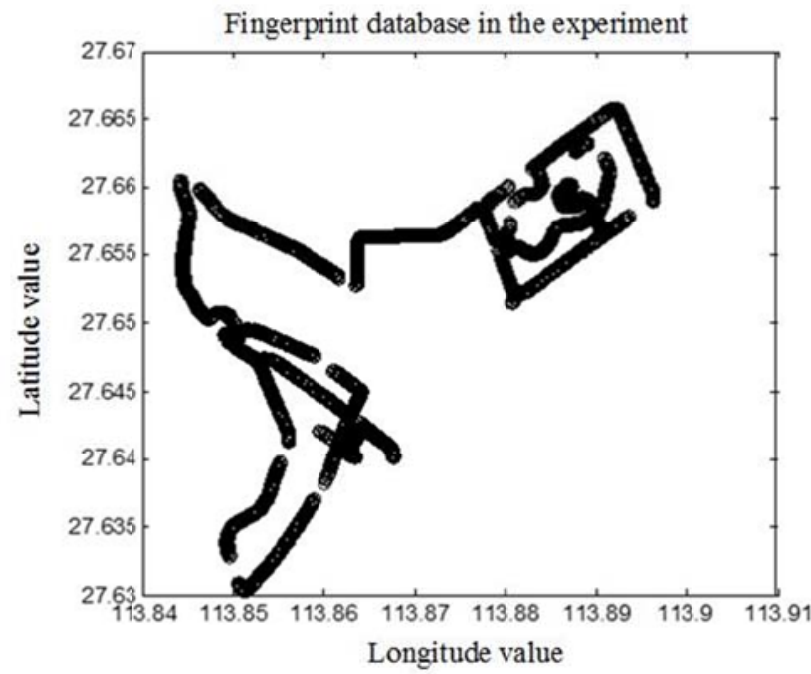

Figure 3 Data used to set up fingerprint database

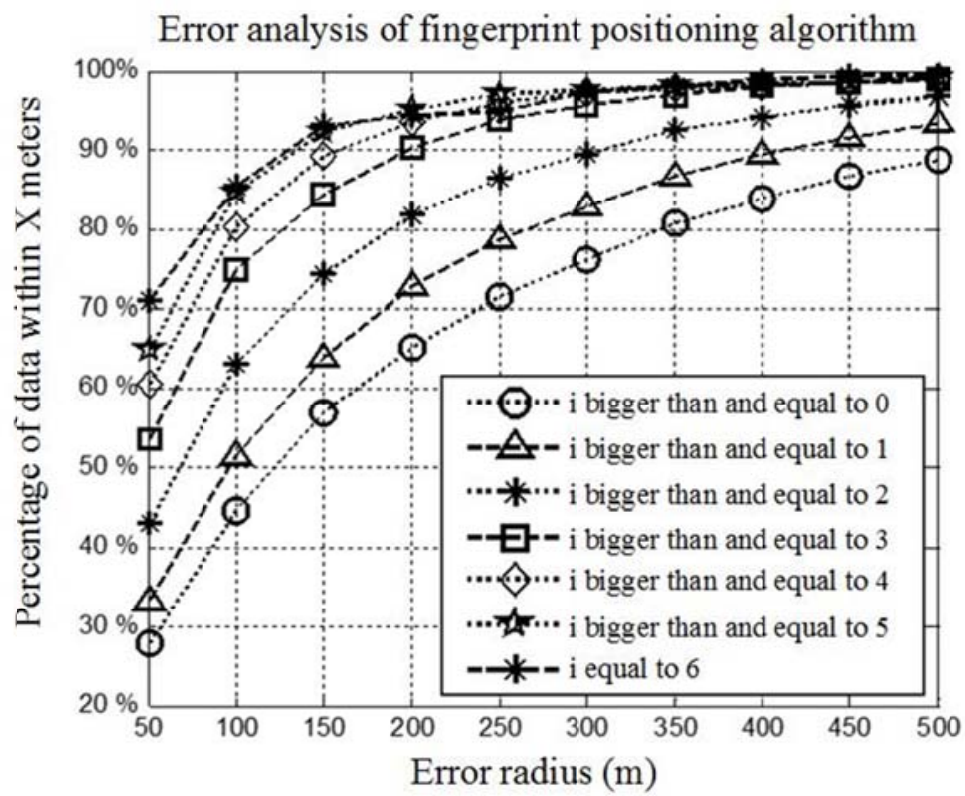

Figure 4 Influence of parameter I selection on fingerprint positioning results based on RSS space time processing

Figure 4 has compared the error result of fingerprint positioning algorithm based on RSS space time processing when the value of parameter I changes. It can be learnt from simulation result that when select the same main service cell (I is bigger than or equal to 0 ) and without considering about neighboring cell, the accuracy can reach $67 \%$ within 200 meters. When the number I of the same neighboring cell is bigger than or equal to 4 , the accuracy rate can reach $67 \%$ within 100 meters. Compared with the algorithms in literatures [2, 3], it avoids complicated algorithm while meeting the same accuracy.

\section{Conclusions}

Adaptive tracking and recording method of dynamic real-time WiFi fingerprint positioning has been studied in this paper; fingerprint database has been set up with spatial segmentation, clustering and temporal filter modifying RSS; fingerprint comparison has been made by compromising the number Euclidean distance equal to neighboring region; fingerprint positioning algorithm whose 
output positioning result is based on RSS space time processing can also be applied to $3 \mathrm{G}$ and LTE and other systems with cellular network structure, but fingerprint database changes with the change of environment. This algorithm makes uses of actual wireless environment to set up fingerprint database, which can satisfy actual positioning without increasing burden for the system and avoiding the complexity of algorithm.

\section{Reference}

[1] Ma R, Guo Q, Hu C, et al. An Improved WiFi Indoor Positioning Algorithm by Weighted Fusion[J]. Sensors, 2015, 15(9):21824-43.

[2] Jin M, Koo B, Lee S, et al. IMU-assisted nearest neighbor selection for real-time WiFi fingerprinting positioning[C]// International Conference on Indoor Positioning and Indoor Navigation. 2014:102-116.

[3] Willaredt J. WiFi and Cell-ID based positioning -Protocols, Standards and Solutions[J]. Snet Project Wt, 2011.

[4] Bisio I, Cerruti M, Lavagetto F, et al. A Trainingless WiFi Fingerprint Positioning Approach Over Mobile Devices[J]. IEEE Antennas \& Wireless Propagation Letters, 2014, 13(1):832-835.

[5] Zhu Y Q, Ou D X, Shen T. Online adaptive positioning algorithm for public location services in indoor places[C]// IEEE, International Conference on Intelligent Transportation Systems. 2014:1515-1520. 